\title{
Statistical mechanics in a discrete space-time. Thermodynamics and time-irreversibility
}

\author{
J.P.Badiali \\ LECA, ENSCP-Université Pierre et Marie Curie, \\ 4 Place Jussieu, 75230 Paris Cedex 05, France
}

Received April 29, 2003, in final form July 21, 2003

\begin{abstract}
The introduction of a discrete space-time represents an attempt to describe the physics at the Planck's scale. We show that this concept can be also very useful in describing thermodynamics in a pre-relativistic world. From this concept a new approach of statistical mechanics based on a dynamic viewpoint and an entropy representation is presented. The entropy is connected with the counting of the paths in space-time. It contains a time interval that represents the time that we have to wait in order to relax the quantum fluctuations and to reach the thermal regime. It is shown that this time is $\beta \hbar$. The mathematical expressions we derive for thermal quantities like the entropy and the free energy are identical to those obtained by the traditional path-integral formalism starting from the canonical form of the thermal density matrix. However, the introduction of a quantized spacetime shows that thermodynamics is consistent with an equation of motion that is time-irreversible at a microscopic level. As a consequence, the problem of irreversibility is revisited and the derivation of a $\mathrm{H}$-theorem becomes possible in the future.
\end{abstract}

Key words: statistical mechanics, thermodynamics, time-irreversibility, discrete space-time

PACS: 03.65.Ca, 05.30.-d, 05.70.-a, 47.53.+n

\section{Introduction}

It is a great pleasure for me to dedicate this paper to my friend Professor Myroslav Holovko for his 60th birthday celebration. His contribution to statistical mechanics and liquid state theory is important and, in parallel, the recent progresses obtained by his former students in Lviv as well as in other countries across the world illustrate the importance of the Ukrainian school in statistical mechanics.

Despite the large successes in statistical physics obtained during the last century some basic problems remain unsolved. The existence and the demonstration of the $\mathrm{H}$-theorem is one of them. Another problem concerns our real understanding of the 
black hole entropy; here we have to deal with the consistency between quantum field theory and general relativity, or the so-called quantum gravitation problem. This paper is an attempt to progress in the derivation of a H-theorem by using ingredients developed in the domain of quantum gravitation.

It is well known that the canonical quantum gravitation theory leads to some infinities that we cannot renormalize. This suggests the introduction of new descriptions for the universe at the Planck's scale. One possible choice consists in abandoning the concept of point particles in quantum field theory and in dealing with superstring theories [1]. Another possibility is to replace the differentiable manifold of the general relativity by a discrete space-time structure $[2,3]$. In this paper we focus on the second alternative and we show that the existence of a discrete spacetime may be a useful concept to describe the physical world at a scale much larger than the Planck's scale. More precisely, we want to show that the thermodynamics of equilibrium is consistent with statistical mechanics in which there exists a motion that is time-irreversible at the microscopic level. This leads to the development of a new viewpoint concerning the problem of the arrow of time in thermodynamics (for a review in this field see for instance [4]).

In the Clausius version, the second law of thermodynamics asserts the existence of a state function, i.e., the entropy, that is a non-decreasing function of time for any closed system [5]. The difficulty in the derivation of this law is the inadequacy between the postulated time-reversible behavior for the motion of particles at a microscopic level and the observable irreversible behavior of macroscopic systems. An illustration of this is the problem of the Poincaré recurrence time associated with the existence of an Hamiltonian [4]. In what follows the motion we consider does not result from a given Hamiltonian but is the consequence of discreteness of the space-time. It is due to this that the problem of the arrow of time is revisited.

More than thirty years ago Feynman presented some doubts concerning the continuum nature of space-time. In a seminal paper Finkelstein [2] has presented the dilemma of the discrete versus the continuous space-time structure, a problem already considered by Riemann in the classical world. Finkelstein, after some others, developed the idea that the topology of space-time is determined by an order structure rather than by a metric structure. Later, the idea of a discrete space-time has been extensively developed via the concept of causal sets [3] for which there are two basic ingredients: the discreteness of space-time and the existence of a causal structure. In parallel to this causal sets theory, in the domain of string theory, a minimal length uncertainty relation related to the fact that strings cannot probe distances below the string scale $[6,7]$ is introduced. Thus, the existence of a minimal length appears to be a more and more common idea today.

Hereafter, a discrete space-time means that any length is built up from a finite number of elementary lengths $\Delta x$ and that the flow of time occurs in a series of individual "ticks" of duration $\Delta t$ [8]. If we accept the existence of a discrete spacetime we have to decide how it is organized [9]. A quite natural assumption is that some basic ingredients of the discrete space-time organization are already familiar to us from our study of the macroscopic world. Hereafter, the problem is that at the 
level of the discrete space-time we have to introduce the ingredients we need from the quantum theory in order to derive standard thermodynamics. In what follows, we define the structure of space-time by a relation between space and time that mimics the uncertainty relations. This seems a quite natural choice because these relations are the basic ingredient on which the quantum mechanics is built up. As we shall see the space-time structure induces a given motion and we may define some quantities as an average over the paths. These averages can be associated with thermodynamic properties. An entropy associated with the space-time structure is introduced but the counting of equilibrium states that appears in a standard approach is now replaced by a counting over the paths in space-time. The tools on which our new approach of statistical physics is based are the path-integral formalism [10] and the entropy representation of statistical mechanics [11].

In section 2 we define the quantized space-time and we describe the motion associated with it. In section 3 we characterize this space-time by counting the number of closed paths associated with a given time interval $\tau$. For a given value of the internal energy $U$ we define an entropy on the space-time. In section 4 we derive the value of $\tau$ and we show that our dynamic approach gives the same mathematical expressions as the ones obtained by Feynman and Hibbs [10]. However, our results have a different physical meaning and they show the consistency between equilibrium thermodynamics and a motion that is time-irreversible at the microscopic level. This result is also discussed in section 4 . In the last section we give a short conclusion and present some new perspectives.

\section{Introduction of a discrete and quantized space-time}

We first consider the case of a point particle of mass $m$. We assume that $m$ induces a discrete structure in space and time. As discussed in the Introduction, this means that the space-time is formed by a set of points $\left(x_{i}, t_{i}\right)$. In this first attempt, in order to be illustrative we assume that the points are located on the sites of a regular lattice. Moreover, we assume that the lattice spacings $\Delta x$ for the spatial coordinates and $\Delta t$ for the time axis are not independent. At this level of description the only relation that we can find between $\Delta x$ and $\Delta t$ is $(\Delta x)^{2} / \Delta t=\hbar / m$.

Whatever the values of $\Delta x$ and $\Delta t$ we immediately have

$$
\Delta x \Delta p=\hbar \quad \text { and } \quad \Delta t \Delta E=\hbar / 2
$$

provided we use $\Delta p=m(\Delta x / \Delta t)$ and $\Delta E=(1 / 2) m(\Delta x / \Delta t)^{2}$. The relations (1) mimic the Heisenberg uncertainty relations but they result from the discreteness of the space-time. Note that these two relations appear here on the same footing as the consequence of $(\Delta x)^{2} / \Delta t=\hbar / m$. In standard quantum mechanics the positionmomentum and the time-energy uncertainty relations are not of the same nature since the second one is not connected with the non-commutation of two operators $[12]$.

By definition, a path corresponds to a set of sites $\left(x_{i}, t_{i}\right)$. We only retain the paths for which the values of $t_{i}$ form a sequence of ordered numbers in such a way 
that $t_{i+1}>t_{i}$ whatever $i$. There is no such a constraint for the coordinate positions $x_{i}$ but we consider that $x_{i+1}$ is necessarily one of the nearest neighbours of $x_{i}$. Thus, to any path there is associated a random walk in the usual sense [13]. In principle, in order to describe the properties of paths we have to work with finite topological spaces as developed in [9] and we also have to find an alike correspondence principle showing in which limit the discrete space-time may appear as a continuum manifold as shown in [14], for instance. Fortunately, our problem is more simple here since we do not focus on a truly relativistic system.

The relation $(\Delta x)^{2} / \Delta t=\hbar / m$ is essential in what follows, but the precise values of $\Delta x$ and $\Delta t$ are not fixed by the uncertainty relations (1). In the pre-relativistic domain the velocity of light goes to infinity. Accordingly, the Compton wavelength goes to zero and no particles can be created by quantum fluctuations. We may assume that both $\Delta x$ and $\Delta t$ tend to zero provided we keep the relation $(\Delta x)^{2} / \Delta t=\hbar / m$. In such conditions the random walk leads to a continuous diffusion process [13] for which the diffusion coefficient, $D=\hbar / 2 m$, has a pure quantum origin. This random walk can be characterized by a continuous function, $q\left(t_{0}, x_{0} ; t, x\right)$ representing the density of transition probability to go from the initial space-time point $\left(t_{0}, x_{0}\right)$ to $(t, x)$ provided that $t \geqslant t_{0}$. From $q\left(t_{0}, x_{0} ; t, x\right)$ and a real-valued function $\phi_{0}(x)$ defined for $\left(t=t_{0}\right)$ we may form a real-valued function $\phi(t, x)$ according to

$$
\phi(t, x)=\int \phi_{0}(y) q\left(t_{0}, y ; t, x\right) \mathrm{d} y
$$

which is the solution of the diffusion equation

$$
-\partial \phi(t, x) / \partial t+D \Delta_{x} \phi(t, x)=0
$$

verifying the initial-value problem $\phi\left(t_{0}, x\right)=\phi_{0}(x)$. In (3) $\Delta_{x}$ means the Laplacian operator taken at the point $x$.

In the presence of an external potential, $u(t, x)$, we generalize (3) into

$$
-\partial \phi(t, x) / \partial t+D \Delta \phi(t, x)-\frac{1}{\hbar} u(t, x) \phi(t, x)=0
$$

In contrast to the case of free diffusion (3), the fundamental solution of (4) cannot be normalized in general [15]. Thus, $q\left(t_{0}, x_{0} ; t, x\right)$ is no more a density of transition probability but it verifies the Chapman-Kolmogorov law of composition [15]

$$
q\left(t_{1}, x_{1} ; t_{2}, x_{2}\right)=\int \mathrm{d} x_{3} q\left(t_{1}, x_{1} ; t_{3}, x_{3}\right) q\left(t_{3}, x_{3} ; t_{2}, x_{2}\right)
$$

provided $t_{1}<t_{3}<t_{2}$ and, therefore, it can be used to describe the transitions in space-time. The evolution in space-time is then represented by a semi-group.

At this point we have to note several important points. The equation (3) has the classical form of a diffusion equation but the diffusion coefficient $D$ has a pure quantum origin $D \rightarrow 0$ if $\hbar \rightarrow 0$. Up to now we consider the paths as the trace of a motion in real time, this motion is induced by the space-time underlying structure 
and it is not connected with a given Hamiltonian. The function $\phi(t, x)$ is a realvalued function. Therefore, $\phi(t, x)$ is not a wave function and it does not have to verify additional constraints. For example, it does not have to be square integrable. Finally $\phi(t, x)$ is defined for $t \geqslant t_{0}$ and the equation (3) is time-irreversible.

By using the Feynman-Kac formula, the fundamental solution of (4) can be written in terms of path integral. Then $q\left(t_{0}, x_{0} ; t, x\right)$ appears as a weighted sum of all the paths $x(t)$ connecting the points $x_{0}$ to $x$ during a time interval $\left(t-t_{0}\right)$; the weight of a path is determined by

$$
A\left[x(t) ; t, t_{0}\right]=\int_{t_{0}}^{t}\left[\frac{1}{2} m\left(\frac{\mathrm{d} x\left(t^{\prime}\right)}{\mathrm{d} t^{\prime}}\right)^{2}+u\left(t^{\prime}, x\left(t^{\prime}\right)\right)\right] \mathrm{d} t^{\prime}
$$

and we have

$$
q\left(t_{0}, x_{0} ; t, x\right)=\int \mathrm{D} x(t) \exp \left\{-\frac{1}{\hbar} A\left[x(t) ; t, t_{0}\right]\right\} .
$$

We may observe that the integrand in (6) looks like the Hamiltonian for a free particle in an external potential. Therefore, $A\left[x(t) ; t, t_{0}\right]$ can be called the Hamiltonian action or, in quantum field theory, the Euclidean action. However, $A\left[x(t) ; t, t_{0}\right]$ is a formal writing [16]. To calculate the path integral we have to discretize $A\left[x(t) ; t, t_{0}\right]$ and the paths that contribute to the path integral are those for which there is nowhere a derivative i.e. no velocity in the usual sense $[17,18]$.

\section{Entropy in the space-time}

On the space-time introduced in the previous section we may define some functions provided we are able to associate to each of them the time on which we have to observe the motion. Amongst all the possible ways of characterizing the space-time we would like to consider the one which is as close as possible from the standard thermodynamics.

For equilibrium states the external potential $u(t, x)$ is not an explicit function of time and as a consequence $A\left[x(t) ; t, t_{0}\right]$ becomes a function of $\left(t-t_{0}\right)$ that we note $A\left[x(t) ; t-t_{0}\right]$. Let us consider a given space-time point $\left(t_{0}, x_{0}\right)$. A way to characterize this point is to count the number of closed paths around it; we note $A_{0}\left[x(t) ; t-t_{0}\right]$ the corresponding Euclidean action. If there is only one closed path around $\left(t_{0}, x_{0}\right)$ we may conclude that there is a strict order around this point. Of course, this is a limiting case in which all the fluctuations around this path should be forbidden. For actual systems we may choose a mean path selected from (6) by using, for instance, the steepest descent method but this path does not realize all the physical content of (6), we also have to consider the fluctuations around this mean path [10]. The larger is this number of paths the larger are the acceptable fluctuations around the mean path and the less defined is the structure around $\left(x_{0}, t_{0}\right)$.

In the spirit of thermodynamics we may consider that the system in the presence of an external field is constrained from its preparation. For instance, we can prepare the system in such a way that it has a precise value for the internal energy $U$. In 
parallel to the Euclidean action $A_{0}\left[x(t) ; t-t_{0}\right]$ we define a thermal action according to $\left(t-t_{0}\right) U$ and we can count the paths for which the Euclidean action does not deviate too much from the thermal action. This leads to weighting the paths using $\left[A_{0}\left[x(t) ; t-t_{0}\right]-\left(t-t_{0}\right) U\right]$ instead of $A_{0}\left[x(t) ; t-t_{0}\right]$. Let us define the quantity

$$
\left.\Gamma\left(t-t_{0}\right)=\int \mathrm{d} x_{0} \int \mathrm{D} x(t) \exp \left\{-\frac{1}{\hbar} A_{o}\left[x(t) ;\left(t-t_{0}\right)\right]-\left(t-t_{0}\right) U\right]\right\} .
$$

In order to associate $\Gamma\left(t-t_{0}\right)$ with a thermal quantity we assume that there is a characteristic time interval, $\tau$, associated with thermodynamics and on which we have to count the paths to be in thermal equilibrium. By analogy with standard thermodynamics we define an entropy for the paths, $S_{\text {path }}$, according to

$$
S_{\text {path }}=k_{\mathrm{B}} \ln \Gamma(\tau)=k_{\mathrm{B}} \ln \int \mathrm{d} x_{0} \int \mathrm{D} x(t) \exp \left\{-\frac{1}{\hbar}\left[A_{o}[x(t) ; \tau]-\tau U\right]\right\} .
$$

This entropy is the starting point on which our statistical mechanics will be based.

The definition of entropy via (9) requires some comments. In (9), the internal energy, $U$, is strictly fixed via the mode of preparation and there is no temperature but a dynamic quantity, $\tau$. Because there is no Gibbs ensemble behind our definition of entropy we cannot relate (9) to canonical or micro-canonical representation of statistical mechanics. In fact, (9) is an entropy representation of statistical mechanics as defined by Callen [11]. Note that the Planck derivation of the black body radiation law is based on such an entropy representation. However, the difference between (9) and the Planck derivation is that we count paths in space-time instead of equilibrium states. The fact that the temperature is not explicit in (9) does not imply that the system is without temperature, we may define a temperature $T_{\text {path }}$ according to the usual thermodynamic relation

$$
\frac{\mathrm{d} S_{\mathrm{path}}}{\mathrm{d} U}=\frac{1}{T_{\text {path }}}
$$

Our definition of the entropy based on dynamic processes in space-time contains $\tau$, which has no equivalent in a standard approach. This time interval appears more explicitly if we rewrite $S_{\text {path }}$ according to

$$
S_{\text {path }}=\frac{k_{\mathrm{B}} \tau}{\hbar} U+k_{\mathrm{B}} \ln Z_{\text {path }}
$$

with

$$
Z_{\text {path }}=\int \mathrm{d} x_{0} \int \mathrm{D} x(t) \exp \left\{-\frac{1}{\hbar} q A_{o}[x(t) ; \tau]\right\}=\int \mathrm{d} x_{0} q\left(0, x_{0} ; \tau, x_{0}\right)
$$

in which we have taken $t_{0}=0$. We may interpret $Z_{\text {path }}$ as the total number of closed paths that we may count during $\tau$, and $q\left(0, x_{0} ; \tau, x_{0}\right)$ is the fundamental solution of (4) associated with closed paths and a time interval $\tau$. Note that $Z_{\text {path }}$ depends on $\tau$ but not explicitly on $U$ in contrast with $S_{\text {path }}$, which is a function of $U$ and $\tau$. 
However, from the usual thermodynamics and also from the invariance properties of the entropy we know that $S$ must depend on $U$ only [19] and the same is expected for $S_{\text {path }}$. Therefore, $U$ and $\tau$ are not independent.

From (11) and (10) we immediately have

$$
\frac{1}{T_{\text {path }}}=\frac{k_{\mathrm{B}} \tau}{\hbar}+\frac{k_{\mathrm{B}}}{\hbar}\left(U+\hbar \frac{\mathrm{d}}{\mathrm{d} \tau} \ln Z_{\text {path }}\right) \frac{\mathrm{d} \tau}{\mathrm{d} U} .
$$

In standard thermodynamics the relation (10) shows that a specific system in which the total internal energy $U$ is fixed will take a given temperature. Here, to use (10) we see from (13) that we have to give a meaning to $\mathrm{d} / \mathrm{d} \tau \ln Z_{\text {path. }}$. In order to do that we use the expression of $Z_{\text {path }}$ in terms of $q\left(0, x_{0} ; \tau, x_{0}\right)$ given in (12). From the Chapman-Kolmogorov law of composition we may write

$$
q\left(0, x_{0} ; \tau, x_{0}\right)=\int \mathrm{d} x_{b} q\left(0, x_{0} ; \delta t, x_{b}\right) q\left(\delta t, x_{b} ; \tau-\delta t, x_{0}\right)
$$

which is true for any $\delta t$ such as $0<\delta t<\tau$. From (12) and (14) we deduce

$$
\hbar \frac{\mathrm{d}}{\mathrm{d} \tau} \ln Z_{\text {path }}=\frac{\hbar}{Z_{\text {path }}} \int \mathrm{d} x_{0} \int \mathrm{d} x_{b} q\left(0, x_{a} ; \delta t, x_{b}\right) \frac{\mathrm{d}}{\mathrm{d} \tau} q\left(\delta t, x_{b} ; \tau-\delta t, x_{0}\right)
$$

which we can rewrite by using (4)

$\hbar \frac{\mathrm{d}}{\mathrm{d} \tau} \ln Z_{\text {path }}=\frac{\hbar}{Z_{\text {path }}} \int \mathrm{d} x_{0} \int \mathrm{d} x_{b} q\left(0, x_{0} ; \delta t, x_{b}\right)\left[D \Delta_{x_{0}}-\frac{1}{\hbar} u\left(x_{a}\right)\right] q\left(\delta t, x_{b} ; \tau-\delta t, x_{0}\right)$.

In this equation the term associated with the external potential can be written

$$
\frac{1}{Z_{\text {path }}} \int \mathrm{d} x_{0} u\left(x_{0}\right) q\left(0, x_{0} ; \tau, x_{0}\right)=\int \mathrm{d} x_{0}\left\langle u_{\mathrm{P}}\left(x_{0}\right)\right\rangle_{\text {path }}
$$

in which we may interpret $\left\langle u_{\mathrm{P}}\left(x_{0}\right)\right\rangle_{\text {path }}$ as the potential energy at the point $x_{0}$ calculated as an average over the paths. To calculate the contribution of the Laplacian in (16) we may use the fact that (14) holds if $\delta t \rightarrow 0$. In that case the Laplacian operates only on the term which looks like the kinetic energy in $A_{o}[x(t) ; \tau]$. A simple calculation shows that this term is

$$
\frac{1}{Z_{\text {path }}} \int \mathrm{d} x_{0} \int \mathrm{d}(\delta x) q\left(0, x_{0} ; \delta t, x_{0}+\delta x\right)\left[\frac{m}{2}\left(\frac{\delta x}{\delta t}\right)^{2}-\frac{\hbar}{2 \delta t}\right] q\left(\delta t, x_{0}+\delta x ; \tau-\delta t, x_{0}\right)
$$

which we can rewrite as

$$
\int \mathrm{d} x_{a}\left[\frac{m}{2}\left\langle\left(\frac{\delta x}{\delta t}\right)^{2}\right\rangle_{\text {path }}-\frac{\hbar}{2 \delta t}\right] .
$$

This expression represents the difference between the kinetic energy calculated as an average over the paths and the quantum fluctuation of energy corresponding to a 
time interval $\delta t$ on which the kinetic energy is calculated. In the limits $\delta x \rightarrow 0$ and $\delta t \rightarrow 0$ we may replace $q\left(0, x_{0} ; \delta t, x_{0}+\delta x\right)$ in (18) by the free particle approximation which only depends on $\delta t$ and $\delta x$. Moreover, we may approximate $q\left(\delta t, x_{0}+\delta x ; \tau-\right.$ $\left.\delta t, x_{0}\right)$ by $q\left(0, x_{0} ; \tau, x_{0}\right)$ and its integral over $x_{0}$ gives $Z_{\text {path }}$ as shown in (12). In the limits that we consider it is easy to see that $m / 2\left\langle(\delta x / \delta t)^{2}\right\rangle_{\text {path }}=\hbar / 2 \delta t$. We may also verify this result by performing an exact calculation but using an explicit form of $u(x)$. Note that such a result is also expected from $(1)$; when $\delta t \rightarrow 0$ we must recover the results of the initial lattice on which we have immediately $1 / 2 m(\delta x / \delta t)^{2}=\hbar / 2 \delta t$ Thus, the term in brackets in (19) is well defined in the limit $\delta t \rightarrow 0$. More generally, we write

$$
\frac{m}{2}\left\langle\left(\frac{\delta x}{\delta t}\right)^{2}\right\rangle_{\mathrm{path}}=\frac{\hbar}{2 \delta t}-\left\langle u_{\mathrm{K}}\left(x_{0}\right)\right\rangle_{\mathrm{path}}
$$

in which $\left\langle u_{\mathrm{K}}\left(x_{0}\right)\right\rangle_{\text {path }}$ is a well behaved function in the limit $\delta t \rightarrow 0$. In principle $\left\langle u_{\mathrm{K}}\left(x_{0}\right)\right\rangle_{\text {path }}$ can be calculated for a given external potential $u(x)$. For instance, in the case of quantum oscillators of frequency $\omega$ we have

$$
\int \mathrm{d} x_{0}\left\langle u_{\mathrm{K}}\left(x_{0}\right)\right\rangle_{\mathrm{path}}=\frac{\hbar \omega}{4}\left(\frac{2}{\exp \tau \omega-1}+1\right) .
$$

In that case this integral is identical to the integral of $\left\langle u_{\mathrm{P}}\left(x_{0}\right)\right\rangle_{\text {path }}$ defined in (17). Finally we can rewrite (13) according to

$$
\frac{\hbar}{k_{\mathrm{B}} T_{\mathrm{path}}}=\tau+\left[U-\int \mathrm{d} x_{0}\left(\left\langle u_{\mathrm{K}}\left(x_{0}\right)\right\rangle_{\mathrm{path}}+\left\langle u_{\mathrm{P}}\left(x_{0}\right)\right\rangle_{\mathrm{path}}\right)\right] \frac{\mathrm{d} \tau}{\mathrm{d} U} .
$$

This equation is the main result of this section. It establishes a relation between $U$, $\tau$, several quantities calculated over the paths and $T_{\text {path }}$.

\section{Thermodynamics and characteristic time}

Up to now we have defined some quantities like entropy, energies and temperature that are similar to the ones defined in standard thermodynamics. However, instead of considering equilibrium states we investigate the properties of closed paths. A dynamic point of view has been developed in which there exists a characteristic time, $\tau$, defined by (22). Now we claim that the value of $\tau$ is such that we are able to recover the standard thermodynamics. This means that all the quantities with the subscript "path" have the same value as in standard thermodynamics although they have a different physical content.

If $S_{\text {path }}$ corresponds to $S$ we may identify $T_{\text {path }}$ and $T$ according to (10). We consider that $\tau$ is such that

$$
\int \mathrm{d} x_{a}\left[\left\langle u_{\mathrm{K}}\left(x_{a}\right)\right\rangle_{\mathrm{path}}+\left\langle u_{\mathrm{P}}\left(x_{a}\right)\right\rangle_{\mathrm{path}}\right]=-\hbar \frac{\mathrm{d}}{\mathrm{d} \tau} \ln Z_{\mathrm{path}}
$$

which looks like the internal energy is indeed equal to the total internal energy of the system energy i.e. $U$. Thus, whatever the value of the derivative $\mathrm{d} \tau / \mathrm{d} U$ from 
(22) we deduce that

$$
\tau=\frac{\hbar}{k_{\mathrm{B}} T}=\beta \hbar
$$

Now, from (23) and (24) we have

$$
\hbar \frac{\mathrm{d}}{\mathrm{d} \tau} \ln Z_{\text {path }}=\frac{\mathrm{d}}{\mathrm{d} \beta} \ln Z_{\text {path }}=-U .
$$

This is the usual relation between internal energy $U$ and the partition function and we have to conclude that $Z_{\text {path }}$ must be identical to $Z$. Using (24) we may rewrite the partition function calculated over paths (12) according to

$$
Z_{\text {path }}=\int \mathrm{d} x_{a} \int \mathrm{D} x(t) \exp -\frac{1}{\hbar} \int_{0}^{\beta \hbar}\left[\frac{1}{2} m\left(\frac{\mathrm{d} x\left(t^{\prime}\right)}{\mathrm{d} t^{\prime}}\right)^{2}+u\left(x\left(t^{\prime}\right)\right)\right] \mathrm{d} t^{\prime} .
$$

We can check that this expression is identical to the one obtained in [10] starting from the canonical form of the density matrix. If we define $F$ according to $F=-k_{\mathrm{B}} T \ln Z$ we see that (11) corresponds to the usual relation $F=U-T S$. Thus, our results obtained from an entropy representation are identical to those derived from the standard canonical formalism. This shows that we have obtained an alternative description of the thermodynamics directly in terms of motion in space-time. In this approach the time is a real time and the motion is not due to a given Hamiltonian but results from the discreteness of the space-time. However, our path integral formalism represents more than a simple alternative description of thermodynamics as we shall see below.

In the standard path integral formalism $\beta \hbar$ is considered to be a formal time [10]. In our approach we have first introduced a given time $\tau$ and, by combining dynamics (4) and thermodynamics via (11) and (10), we have shown that $\tau=\beta \hbar$. Therefore, we may conclude that $\tau$ has a strong physical meaning. A first discussion regarding this point has been already presented in [18]. Here $\tau$ appears to be the relaxation time that we have to wait in order to relax the quantum fluctuations and to reach the thermal regime. From standard textbooks in statistical mechanics [5] it is well known that such a time exists and as a consequence there is no entropy on a short period of time. However this time plays no role in the Gibbs ensemble approach for which there is no dynamics.

However, the most important result of our approach is that equilibrium thermodynamics is consistent with a motion at the microscopic scale which is time-irreversible since given by (4). This consistency can be explained as follows. Let us consider a system at equilibrium with a given entropy $S$ and we investigate its fluctuations starting from an initial time $t=0$. By convention, we can say that the entropy at the initial time is given by $S=k_{\mathrm{B}} \ln \Gamma$ where $\Gamma$ is given by (9). However, to calculate $S$ we see that starting from $t=0$ we have to follow the system during a time interval $\tau$. For $0<t<\tau$ there is no entropy and more generally no thermodynamics since the quantum fluctuations are larger than the standard thermal fluctuations. 
However, the knowledge of the system evolution during this time interval allows us to calculate its entropy. For $0<t<\tau$ we may inspect the system with a time scale $\delta t \ll \tau$. If $\delta t \rightarrow 0$ during the first time interval we have an energy fluctuation $\hbar / 2 \delta t$ that can be much larger than the mean thermal energy $U_{\mathrm{K}}$. Thus, the evolution of the system must be such that the fluctuations relax the energy from a high value, $\hbar / 2 \delta t$, to the mean thermal kinetic energy $U_{\mathrm{K}}$. This is precisely the physical meaning of $(20)$ which we can rewrite as

$$
U_{\mathrm{K}}=\int \mathrm{d} x_{a}\left\langle u_{\mathrm{K}}\left(x_{a}\right)\right\rangle_{\mathrm{path}}=\frac{\hbar}{2 \delta t}-\int \mathrm{d} x_{a} \frac{m}{2}\left\langle\left(\frac{\delta x}{\delta t}\right)^{2}\right\rangle_{\mathrm{path}} .
$$

Hence, the evolution of the system during the time interval $(\tau-\delta t)$ must be oriented and cannot result from a time-reversible motion. Of course, after this relaxation the system recovers its initial equilibrium and there is no net dissipation during the time interval $\tau$. This can be shown from simple arguments in the case of a free particle. By analogy with the case of a moving body [19] between two thermal equilibrium states we can write $\delta S=\delta E-\langle v \delta p\rangle$. This expression relates the mean changes of entropy $\delta S$ and internal energy $\delta E$ to the mean change of the mechanical work $\langle v \delta p\rangle$ where $v$ is the velocity and $p$ is the momentum. We may estimate $\langle v \delta p\rangle$ to be $\delta\left\langle\left(1 / 2 m v^{2}\right)\right\rangle$ and $\delta E$ to be $\delta E=\hbar / 2 \tau=k_{\mathrm{B}} T / 2$. Thus, the absence of dissipation $\delta S=0$ means that the quantum fluctuation is used to give a mean change of kinetic energy $\delta\left\langle\left(1 / 2 m v^{2}\right)\right\rangle$ corresponding exactly to the mean value of the kinetic energy $k_{\mathrm{B}} T / 2$. This shows that the time $\tau$ is such that the mean quantum fluctuation is compensated by the thermal kinetic energy in such a way that there is no entropy change between equilibrium states.

In summary we have shown that in an equilibrium state the motion is not timereversible but appears in a given direction from which we relax the large quantum fluctuations. We may try to extend our approach in order to describe the irreversible evolution of a given system which is not in equilibrium at the initial time. This should lead to a demonstration of a H-theorem. Such a demonstration is possible as it will be shown in a subsequent paper.

\section{Conclusions}

In this paper a new derivation of the path integral formalism of statistical mechanics is presented. Here we give a physical meaning to the paths. They represent some motions in a quantized space-time. These motions do not result from a given Hamiltonian but they are the consequence of the discreteness of the space-time. Focusing on the counting of paths we recover the results obtained in [10] for the partition function and the entropy although our results have a different physical meaning. These results show that the standard thermodynamics is consistent with the motions that are not time-reversible at a microscopic scale. This offers the possibility to derive a H-theorem without a new assumption.

The work presented requires future investigations. Many aspects developed here are taken from the relativity theory. Therefore, it would be nice to elaborate a totally 
covariant theory. In our approach, time-irreversible processes play a dominating role but it would be important to improve the theory in order to create time-reversible processes. Some progresses in these two directions have been already done.

\section{References}

1. Kaku M. Introduction to Superstring. New York, Springer-Verlag, 1990.

2. Finkelstein D. // Phys. Rev., 1969, vol. 184, p. 1261.

3. Bombelli L., Lee J., Mayer D., Sorkin R.D. // Phys. Rev. Lett., 1987, vol. 59, p. 521.

4. Zeh H.D. The Physical Basis of the Direction of Time. Berlin, Springer-Verlag, second edition, 1992.

5. Landau L.D., Lifshitz E.M. Statistical Physics. Oxford, Pergamon Press, 1977.

6. Chang L.N., Minic D., Okamura N., Takeuchi T. // Phys. Rev. D, 2002, vol. 65, p. 125028.

7. Kempf A., Mangano G., Mann R.B. // Phys. Rev. D, 1995, vol. 52, p. 1108.

8. Reid D.D. // Can. J. Phys, 2001, vol. 79, p. 1.

9. Sorkin R.D. // International Journal of Theoretical Physics, 1991, vol. 30, p. 923.

10. Feynman R.P., Hibbs A.R. Quantum Mechanics and Path Integrals. New York, McGraw Hill, 1965.

11. Callen H.B. Thermodynamics and an Introduction to Thermostatistics. New York, John Wiley, 1985.

12. Landau L.D., Lifshitz E.M. Quantum Mechanics. Oxford, Pergamon Press, 1977.

13. Itzykson C., Drouffe J.M. Statistical Field Theory. Cambridge, Cambridge University Press, 1989.

14. Raptis I., Zapatrin R.R. // International Journal of Theoretical Physics, 2000, vol. 39, p. 1.

15. Nagasawa M. Stochastic Processes in Quantum Physics. Monographs in Mathematics, vol. 94. Basel, Birkhauser Verlag, 2000.

16. Wiegel F.W. Introduction to Path Integral Methods in Physics and Polymer Science. Singapore, World Scientific, 1986.

17. Badiali J.P. // Phys. Rev. E, 1999, vol. 60, p. 2533.

18. Badiali J.P. // Condens. Matter Phys., 2000, vol. 3, p. 545.

19. Giles R. Mathematical Foundations of Thermodynamics. International series of monographs on pure and applied mathematics. New York, McMillan, 1964. 
Статистична механіка в дискретному просторі-часі. Термодинаміка і часова необоротність

\author{
Ж.П.Бадіалі \\ Університет ім. П'єра і Марії Кюрі, \\ Франція, 75230 Париж, Площа Жюсс'ї, 4 \\ Отримано 29 квітня 2003 р., в остаточному вигляді - 21 липня \\ 2003 p.
}

Введення дискретного простору-часу $є$ спробою опису фізики в масштабі Планка. Нами буде показано, що така концепція може також бути корисною для опису термодинаміки в пре-релятивістичній системі. Виходячи з цієї концепції, нами запропоновано новий статистико-механічний підхід, що базується на динамічній точці зору i представленні ентропії. Ентропія пов'язана з процедурою розрахунку шляхів в просторі-часі. Вона містить часовий інтервал, що являє собою час необхідний для загасання квантових флуктуацій і досягнення термічного режиму. Показано, що $\beta \hbar є$ цим часом. Математичні вирази, отримані нами для таких термічних величин як ентропія і вільна енергія, є ідентичними з такими, отриманими з традиційного формалізму інтегрування по траекторіях, починаючи з канонічної форми термічної матриці густини. Проте, введення квантованого простору-часу показує, що термодинаміка узгоджується з рівнянням руху, яке $€$ незворотнім в часі на мікроскопічному рівні. Як наслідок, знову постає проблема необоротності і вивід Н-теореми стає можливим у майбутньому.

Ключові слова: статистична механіка, термодинаміка, необоротність часу, дискретний час-простір

PACS: 03.65.Ca, 05.30.-d, 05.70.-a, 47.53.+n 